\title{
P300 characterization using empirical mode decomposition
}

\author{
Victor S. Braz, Ana Claudia S. Souza, and Gustavo F. Rodrigues
}

\begin{abstract}
As the communication between brain and computer becomes more accessible the extraction of important features of electrophysiological signals is an essential step in artificial communication systems. This paper proposes the usage of the Empirical Mode Decomposition to identify characteristics of the P300 signal and classify target and non-target signals using a feedforward neural network. The results show that through the usage of EMD method it is possible to identify the P300 signal using low volume of data.
\end{abstract}

Keywords-brain-computer interface, P300, empirical mode decomposition, intrinsic function

\section{INTRODUCTION}

A brain-computer interface (BCI) is a system capable of translating brain signals onto commands and action. It is usually used by individuals with motor disability as it enables the person to perform a specific task without involving muscular movement [1], [2]. In this system, the so called Event Related Potentials (ERPs) are used as driven signals. A very common event related potential used in the analysis of BCI systems is the P300 evoked potential. P300 potentials are based on the oddball paradigm, which is defined as a random series of event stimuli that contain an infrequently set of items. The P300 is a low frequency signal occurring around $300 \mathrm{~ms}$ after the stimulus onset. This ERP is elicited when a rare (target) event appears in a sequence of regular events [3].

An important step of the ERP detection problem is the feature extraction due to poor signal to noise ratio of a single epoch. The most used method to detect a P300 is the averaging of several epochs that contain the ERP but this can be time consuming which reflects in processing delay in a BCI. Therefore, single trial ERP detection is being widely studied [4]. When studying characteristics of an specific signal a way to understand its basis is through decomposition. There is a vast number of ways to decompose a signal. These methods can have different mathematical or empirical approaches, different complexity and areas of application.

Single trial EEG analysis has been developed for timelocked and phase-locked evoked potentials. Methods include blind source separation [5], Kalman filter [6], wavelet methods [7] among others. However, signal extraction in these methods requires prespecified basis functions.

Manuscript received June 19, 2019; revised December 16, 2019.

Victor S. Braz is with Ericsson Telecomunicações, São Paulo-SP, Brazil, e-mail: (vsoaresbraz@gmail.com).

Ana Claudia S. Souza and Gustavo F. Rodrigues are with Universidade Federal de São João del-Rei, Ouro Branco-MG, Brazil. e-mail: (aclaudia,gfernandes@ufsj.edu.br)
The Empirical Mode Decomposition (EMD) method has particular properties and advantages over these methods. Its basis functions used for decomposition are derived from the data itself and not from basis functions defined a priori such as in the Fourier or Wavelet transform. The Hilbert-Huang transform (HHT) which uses the EMD derives the frequency by differentiation, therefore, the uncertainty principle on time and frequency resolution does not apply [8].

The EMD is a method of analysis that represents a signal as the sum of zero-mean amplitude-frequency modulated signals where each element is known as Intrinsical Mode Function (IMF). As the EMD decomposes the signal based on its own data it can be used to decompose characteristics of EEG signals with $\mathrm{P} 300$. Once a number of features have been extracted for each epoch a neural network is used as a classifier in the detection task.

\section{A. Hilbert Huang transform}

The Hilbert Huang transform (HHT) decomposes a signal using the EMD and calculates its instantaneous frequency spectrum using Hilbert spectrum. It was proposed by Huang in [8] and consists of the decomposition of the signal into a finite number of the so called intrinsic mode functions (IMF) and the Hilbert spectral analysis.

The HHT has a wide range of application for nonlinear and non-stationary time series analysis. It was originally designed to study fluid mechanics [8], but in sequence found applications in different fields such as fault diagnosis [9], data series prediction [10], biomedical signal analysis such as blood pressure [11], elliptic seizure detection and EEG decomposition [12], [13], and analysis of olfactory ERPs [14].

\section{B. Empirical Mode Decomposition}

EMD allows a flexible sub-band signal decomposition while preserving the nonlinear and nonstationary features of the signals which is essential for brain activity analysis. It is an adaptive method, that is, the decomposition it produces is specific to the analyzed signal. It decomposes the signal into a sum of components, each with varying amplitude and phase, and should separate phenomena occurring on different time scales. EMD decomposes the signal into a set of high frequency modes called Intrinsic Mode Functions (IMFs) and a low frequency component called the residual. The method decomposes the original signal as expressed in Equation 1 .

$$
X(t)=\sum_{j=1}^{m} C_{j}(t)+R_{m}(t)
$$


where $X(t)$ is the original signal in time domain, $C_{j}(t)$ is $j^{t h} \mathrm{IMF}$, and $R_{m}(t)$ the residue. Therefore, we can select the IMFs combination to reconstruct the signal of interest and discard the remaining IMFs which contribute to noise.

An IMF has to fulfill two conditions: (i) in the whole data set, the number of extrema and the number of zero crossings must be either equal to or differ by one at most. (ii) the mean value of the envelope defined by the local maxima and minima is zero at any point. These conditions are necessary to define instantaneous frequency.

The starting point of the EMD is to locally estimate a signal as a sum of a local trend i.e. the low frequency content and a local detail i.e. the high frequency content. When this is done for all the oscillations composing a signal, the procedure is then applied again to the residual, considered as a new time series, extracting a new IMF and a new residual. The process continues until IMF satisfies the two criteria specified above. At the end of the decomposition process, the EMD method provides a signal as the sum of a finite number of IMFs and a final residual.

The algorithm can be summarized as follows:

(a) Identify maxima and minima of the signal $X(t)$;

(b) Interpolate between identified points to generate the superior wrap, $e_{\max }(t)$ and the inferior wrap $e_{\min }(t)$;

(c) Compute the mean of the wraps, $m_{1}=\frac{e_{\min }(t)+e_{\max }(t)}{2}$

(d) Subtract the mean from the original signal, $c(t)=X(t)-$ $m(t)$

At this point, $\mathrm{IMF}_{1}=c(t)$ and the residue $r(t)=x(t)-c(t)$ becomes the new input signal for step (a). This process is iterated until the resulting signal $c(t)$ complies with the criteria of an intrinsic mode function.

\section{Application of the HHT to EEG signals}

EMD has been used in the removal of noise in EEG data such as eye blinks and eye movements. It has been used with independent component analysis (ICA) to decompose ICs that correspond to artifacts [17]. It has also been applied in conjunction with wavelet analysis to remove movement-related artifacts from EEG data [18] and with principal component analysis to remove cardiac artifact [19].

The approach has also been applied to identify neural activity sources related to ERPs such as described in Li et al [20] which used EMD to detect disease states following a stroke. EMD has the potential to be used to identify taskrelevant activity from trial to trial in different individuals, because of the adaptive data decomposition it provides. EMD has been implemented to detect neural correlates of movement in EEG recordings [21] and classification in motor imagery tasks [22], [23], [24]. Although most of the potentials used for BCI are movement related some authors report the usage of EMD to steady-state visual evoked potentials (SSVEP) [25]. Although these potentials have stationary frequency, the physiological noise recorded with it is not [26].
EMD also seems to be suitable to study the P300 potential as it is composed by a low frequency component embedded in high frequency oscillations. However, there are very little work showing the implementation of EMD with P300 potentials. Solis-Escarlate et al. [27] used EMD and support vector machines (SVM) for single trial detection of the P300 potential. The authors decomposed an average P300 response and used least-squared of a single EEG epoch to the IMFs.

This paper proposes the usage of the Hilbert Huang transform through the Empirical Mode Decomposition to identify characteristics of the P300 signal and classify target and nontarget signals using a neural network.

\section{Methodology}

\section{A. Dataset}

The dataset II from the Third Edition of the BCI competition was used in this project [28]. This dataset is based on the P300 speller paradigm proposed by Farwell and Donchin [29]. In this paradigm, a $6 \times 6$ matrix with letters and numbers is exhibited to the subject. Columns and rows are highlighted in a frequency of $5,7 \mathrm{~Hz}$ and the subject is asked to focus his attention on specific target characters. The data set contains EEG recordings of two subjects. Each run consists of 64 channels with sampling frequency of $240 \mathrm{~Hz}$ and 85 characters with 15 runs per character. Electrodes $\mathrm{FC} 1, \mathrm{FC} 2$ and $\mathrm{Cz}$ were selected as they are located close to $\mathrm{Cz}$ electrode which, historically, has shown clear P300 signals.

\section{B. EMD for feature extraction}

The EMD algorithm was implemented using Matlab software. The first attempt was to investigate the influence of the number of trials in the averaged signal used in the decomposition. Three configurations were used:

(a) IMFs of the average of 85 characters (15300 epochs)

(b) IMFs of the average of 40 characters (7200 epochs)

(c) IMFs of one character (180 epochs)

\section{Classification}

For P300 classification, a feedforward neural network trained with a resilient backpropagation learning algorithm was used. The structure of the neural network consists of an input layer, two hidden layers with 10 and 5 neurons each and an output layer. In the first and second hidden layers a log-sigmoid activation function was used. The output layer had one neuron and a linear activation function was applied. These values were chosen based on trial and error until performance was no longer improved, as shown in Table I.

The neural network was trained with $65 \%$ of the data set available per subject and $35 \%$ of the data set were used to test and verify the performance of the system. In the training phase, the pattern data is randomly selected for each subject and presented to the input layer and the desired output at the output layer. In the testing phase, $35 \%$ of the whole dataset was evaluated.

In the classification step different procedures were investigated: 
TABLE I: Mean hit rate vs. number of neurons in each layer

\begin{tabular}{c|c|c|c|c}
\hline Layer 1 & Layer 2 & Layer 3 & mean hit rate (\%) & standard deviation \\
\hline 80 & 40 & 1 & 91.2 & \pm 5.5 \\
40 & 20 & 1 & 93.5 & \pm 3.9 \\
20 & 10 & 1 & 95.3 & \pm 1.6 \\
10 & 5 & 1 & 95.3 & \pm 1.1 \\
\hline
\end{tabular}

i Classification using intrinsic mode functions: IMF of each of the three configurations described in $B$ were used as input to the neural network. P300 signals of $\mathrm{Cz}$ electrode were used as target output during training.

ii Classification using original signal:

a average of one epoch of target signals using all 85 characters

b average of all 30 epochs of target signals using one character

c energy of the target signal for each character

d spectrum of the target signal for each character

The neural network input is a vector of 240 elements except for (ii.c) which is a single element.

\section{REsults AND Discussion}

IMF waveforms were obtained for the two subjects. Fig 1 and Fig 2 show the IMFs of target and non-target signals of subjects $\mathrm{A}$ and $\mathrm{B}$ at electrode $\mathrm{Cz}$. Top to bottom IMFs are plotted from highest to lowest frequency. From these figures it is possible to see that IMF labeled $\mathrm{IMF}_{4}$ is a visual candidate to represent $\mathrm{P} 300$ as it peaks from $300 \mathrm{~ms}$ to $500 \mathrm{~ms}$. Moreover, these plots show that IMFs of target and non-target signals are very distinct.
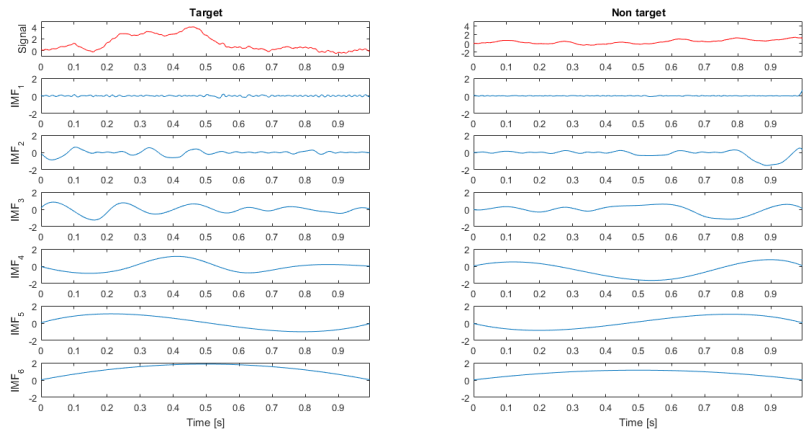

Fig. 1: Subject A intrinsic mode functions of target and non-target signals. Topmost plot depicts original signal while subsequent plots show the decomposition.

\section{A. Target and non-target classification using IMFs}

Since EMD decomposition can capture differences between target and non-target, the IMFs can be used in a classification process. The following analysis consisted in investigating the influence of the number of IMFs in the classification performance. The leftmost column of Table II depicts the number of characters used in the average of the original signal Each character has 30 epochs. Classification accuracy superior than $90 \%$ was achieved using one of the first 4 IMFs which
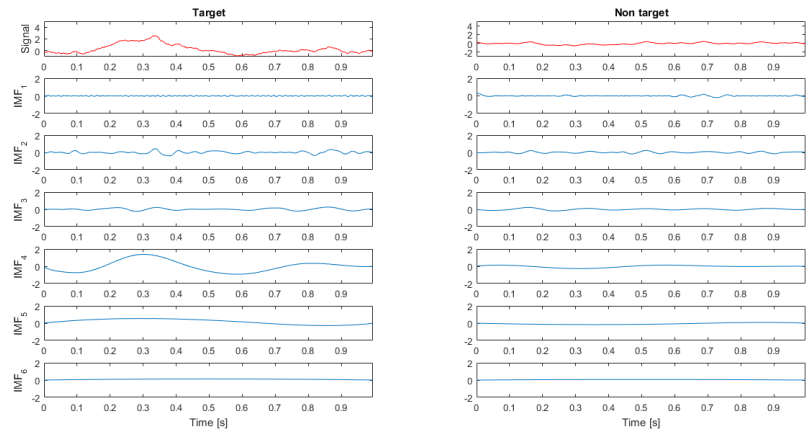

Fig. 2: Subject B intrinsic mode functions of target and non-target signals. Topmost plot depicts original signal while subsequent plots show the decomposition.

probably has meaningful content as hit rate drops considerably with IMFs 5 and 6. Table [II] shows the influence of IMF in the classification hit rate. It is clear that with only one IMF the neural network was able to classify target and non-target signals. This result is particularly interesting if we consider that even when using a small number of characters the IMF is able to capture important characteristics of the signal that will distinguish target from non-target. Futhermore, from Fig 1 and Fid2 it is possible to infer that the lower IMFs are very similar in target and non-target signals as they represent very low frequency, such as the trend of the signal.

TABLE II: Mean hit rate (\%) vs. number of characters and IMF number

\begin{tabular}{c|c|c|c|c|c|c}
\hline Character & IMF 1 & IMF 2 & IMF 3 & IMF 4 & IMF 5 & IMF 6 \\
\hline 1 & 100 & 100 & 100 & 95 & 5 & 0 \\
40 & 100 & 100 & 5 & 0 & 0 & 0 \\
85 & 100 & 95 & 5 & 0 & 0 & 0 \\
\hline
\end{tabular}

In order to compare the classification results obtained using EMD, target and non-target original data was classified using 4 different approaches: average signal of 85 characters (1 epoch of each character), average signal of 30 epochs of one character, energy of the signal and spectrum of the signal as described in section C. Fig 3 shows the hit rate obtained with each of these approaches for both subjects. Best accuracy was achieved when using the energy of the signal as the input to the neural network $(96.5 \%$ hit rate for subject A and $93.0 \%$ hit rate for subject B). This figure also shows that using various epochs from the same character leads to better results than using the same epoch but from different characters even if the latest implies in more trials being averaged (II and I in Fig 3). This result is particularly interesting as one would expect that the more characters are used on the grand average of a P300 signal the better the classification accuracy. However, it is possible that using epochs from the same character in an average brings more consistency to the signal representation than using random characters. The same logic applies to Table II when using 85 characters in the IMF decomposition. IMF4 which visually matches with a P300 representation doesn't add information to the classification. Although an interesting result it deserves better investigation.

Table III summarizes the methods used in this work with the 


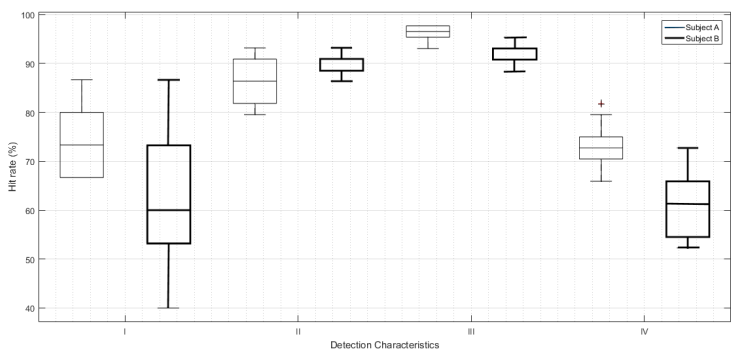

Fig. 3: Classification using original data for subjects A and B. I) average of target signals of all 85 characters using one epoch each. II) average of target signals of all 30 epochs for one character. III) energy of the target signal for each character. IV) spectrum of a target signal for each character.

classification hit rate achieved as well as the number of trials necessary to achieve this hit rate. The method implemented in this work achieves superior performance without the need of averaging an extensive number of trials.

TABLE III: Classification methods

\begin{tabular}{c|c|c|c}
\hline Method & condition & hit rate $(\%)$ & data volume \\
\hline Experiment I & 1st IMF & 100 & low (180 epochs) \\
\hline \multirow{3}{*}{ Experiment II } & II.a & 73.8 & \\
& II.b & 88.2 & high (15300 epochs) \\
& II.c & 96.5 & \\
& II.d & 72.9 & \\
\hline
\end{tabular}

\section{CONCLUSION}

This work presents the usage of empirical mode decomposition (EMD) in processing EEG signals containing P300 evoked potential. The result of achieving a high hit rate ( $>95 \%$ ) without averaging the whole signal is promising since single trial classification can be used in online processing of BCI applications. Further work should consider processing the decomposed signals, the intrinsic mode functions such as extracting its frequency content to identify relevant features for the P300 signal.

\section{ACKNOWLEDGMENT}

The authors would like to thank Fapemig and CNPq for the financial support.

\section{REFERENCES}

[1] N. Birbaumer. Breaking the silence: brain-computer interfaces (BCI) for communication and motor control. Psychophysiology, 43:517-532, 2006.

[2] H. Hwang, K. Kwon and C. Im. Neurofeedback-based motor imagery training for brain-computer interface (BCI). Journal of Neuroscience Methods, 179:[1]:150-156,2009.

[3] A. Furdea, S. Halder, D. Krusienski, D. Bross, F. Nijboer, N. Birbaumer, and A. Kubler, An auditory oddball (p300) spelling system for braincomputer interfaces, Psychophysiology, vol. 46.3, pp. 617625, 2009.

[4] N. Haghighatpanah, R. Amirfattahi, V. Abootalebi, B. Nazari. A single channel-single trial P300 detection algorithm, IEEE 21st Iranian Conference on Electrical Engineering, 2013.

[5] G. Barbati, R. Sigismondi, F. Zappasodi F. Functional source separation from magnetoencephalographic signals . Hum Brain Mapp, 27:925934, 2006. doi:10.1002/hbm.20232.

[6] L. Daubigney, O. Pietquin. Single-trial P300 detection with Kalman filtering and SVMs, in ESANN, 2011.
[7] J. Spinnato, M. Roubaud, B. Burle and B. Torresani. Detecting single-trial EEG evoked potential using a wavelet domain linear mixedl model: application to error potentials classification. Journal of Neural Engineering, 12[3],2015.

[8] N. Huang, Z. Shen, S. Long, M. Wu, S. Shih, Q. Zheng, N. Yen, C. Tung and $\mathrm{H}$. Liu. The empirical mode decomposition and the hilbert spectrum for nonlinear and non-stationary time series analysis. Proceedings of the Royal Society A: Mathematical, Physical and Engineering Sciences[454] 1998

[9] L. Qu and F. Wu. An improved method for restraining the end effect in empirical mode decomposition and its applications to the fault diagnosis of large rotating machinery. Journal of Sound and Vibration, 314:586602, 2008.

[10] G. Oladosu. Identifying the oil price-macroeconomy relationship: An empirical mode decomposition analysis of us data. Energy Policy, 37[12]:5417 5426, 2009.

[11] W. Huang, Z. Shen, N. Huang, and Y. Fung. Engineering analysis of biological variables: An example of blood pressure over 1 day Proceedings of the National Academy of Sciences, 95[9]:4816 4821, 1998.

[12] A. Tafreshi, A. Nasrabadi, and A. Omidvarnia. Epileptic seizure detection using empirical mode decomposition. IEEE International Symposium in Signal Processing and Information Technology, 2008. ISSPIT 2008, pages 238 242, 2008

[13] V. Bajaj. EEG signal classification using empirical mode decomposition and support vector machine. Proceedings of the International Conference on Soft Computing for Problem Solving, pages 623-635, 2011.

[14] C. Wu. Empirical Mode Decomposition-Based Approach for Intertrial Analysis of Olfactory Event-Related Potential Features. Chemosensory Perception. 5[3-4]:280-291, 2012

[15] K. Shen, C. Ong, X. Li, Z. Hui, and E. Wilder-Smith. A feature selection method for multilevel mental fatigue EEG classification, IEEE Trans Biomed Eng, 54[ 7]:12311237, 2007.

[16] E. Alharbi, S. Rasheed and S. Buhari. Single trial classification of evoked EEG signals due to RGB colors. 7[1], 2016.

[17] J. Lindsen and J. Bhattacharya. Correction of blink artifacts us ing independent component analysis and empirical mode decomposition. Psychophysiology, 47: 955960, 2007, doi: 10.1111/j.14698986.2010.00995.x

[18] V. Bono, S. Das, W. Jamal and K. MaharatnaHybrid wavelet and EMD/ICA approach for artifact suppression in pervasive EEG. J. Neurosci. Methods, 267: 89107, 2016, doi: 10.1016/j.jneumeth.2016.04.006

[19] E. Javed, I. Faye, A. Malik and J. Abdullah. Removal of BCG artefact from concurrent fMRI-EEG recordings based on EMD and PCA. J. Neurosci. Methods, 291: 150165, 2017, doi: 10.1016/j.jneumeth.2017.08.020

[20] X. Li, Y. Yan and W. Wei. Identifying patients with poststroke mild cognitive impairment by pattern recognition of working memory load-related ERP. Comput. Math. Methods Med., 2013, doi: 10.1155/2013/658501.

[21] C. Sweeney-Reed and S. Nasuto.Detection of neural correlates of self-paced motor activity using empirical mode decomposition phase locking analysis. J. Neurosci. Methods, 184: 5470, 2009, doi: 10.1016/j.jneumeth.2009.07.023

[22] S. Taran, V. Bajaj, D. Sharma, et al. Features based on analytic IMF for classifying motor imagery EEG signals in BCI applications. Measurement, 116: 6876, 2018, doi: 10.1016/j.measurement.2017.10.067.

[23] M. Alam and S. Samanta. Empirical mode decomposition of EEG signals for brain computer interface. In: SoutheastCon 2017. IEEE, pp 16, 2017.

[24] I. Daly, S. Nasuto and K. Warwick. Brain computer interface control via functional connectivity dynamics. Pattern Recogn., 45: 212336, 2011, doi: 10.1016/j.patcog.2011.04.034

[25] C. Wu, H. Chang, P. Lee, et al. Frequency recognition in an SSVEP based brain computer interface using empirical mode decomposition and refined generalized zero-crossing. J. Neurosci. Methods, 196: 17081, 2011, doi: 10.1016/j.jneumeth.2010.12.014.

[26] C. Sweeney-Reed, S. Nasuto, M. Vieira, A. Andrade. Empirical mode decomposition and its extensions applied to EEG analysis: a review. Advances in Data Science and Adaptive Analysis,10[2], 2018.

[27] T. Solis-Escalante, G. Gentiletti and O. Yanez-Suarez. Single trial P300 detection based on empirical mode decomposition. In International Conference of the IEEE Engineering in Medicine and Biology Society, 2006

[28] B. Blankertz. BCI Competition III Webpage. Available Online at: http://www.bbci.de/competition/iii/.

[29] L. Farwell and E. Donchin. Talking off the top of your head: toward a mental prosthesis utilizing event-related brain potential. Electroencephalogr and Clin Neurophy, 70:510-523, 1988. 